\title{
Analysis of the Current Status and Development of Internet+ Medical Care in China
}

\author{
WU Yue, XIA Kaijian
}

Soochow University Changshu Hospital (ChangshouNo.1 people's Hospital), Changshu, Jiangsu 215500, China

Keywords: medical care + internet, mobile medical care, digitize

\begin{abstract}
Objective: To improve the current status of medical care, to achieve a sound pension system, and to provide patients with better quality of medical services. Method: Through cooperation between the government and enterprises, medical care + Internet platform is opened up and information is shared. Result: Medical resources are rationally allocated. Online and offline medical services are combined. Interconnection is strengthened. Disease prevention is achieved. And such aspects as the way to hospitalize and purchase medicine as well as improving relationships between doctors and patients are optimized. Conclusion: Compared with traditional hospitalization experiences, Internet medical care facilitates these. Thus pressure on medical institutions is effectively reduced, service efficiency and patient satisfaction are improved, patients' medical experiences are perfected, so that the national health level is comprehensively enhanced.
\end{abstract}

\section{Introduction}

China is the country which comes first in population worldwide. As time goes on, it will grow into a country with a large aging population in the world. Medical care, pension and other issues seem particularly prominent. It will be an urgent task in the future to speed up the reform of the medical care system and achieve a sound pension system. However, with doctor-patient relationships getting more and more tense and contradicted, problems in the medical system have gradually emerged as a major problem that affects social stability. Therefore, it is imperative to reform the medical system and is necessary to introduce parallel development in multiple channels.

In accordance with the division of the informatization level of foreign medical systems, the development of hospital informatization will go through three stages: hospital information system (HIS) stage, clinical information system (CIS) stage and geographic medical information system (GMIS) stage. China's hospital informatization process started quite late. Some hospitals began the development and application of HIS in the late 1970s. In the 1990s, with the development of computer technology, especially network and multimedia technology and that of hospital informatics, the application of medical information management is getting larger and larger in scale and will make hospitalization more diversified.

Since 2014, investments in the field of Internet medical care have seen increases in various countries worldwide. Investments in the United States increased by 125\%more in 2014than those in 2013, and the amount was the sum of the past three years. China's investments increased by $226 \%$ more than those in 2013, the total was 2.5 times that of the past three years. The main areas of U.S. Internet medical care investment in 2014 were: medical big data analysis, medical consumer services, digitized medical equipment, telemedicine. China's main investment areas included: genetic testing, medicine business, wearable equipment and so on. Internet medical care aims to reduce the over crowdedness in hospitalization in public hospitals in China, to strive to optimize and integrate medical resources, and to improve hospitalization efficiency. 


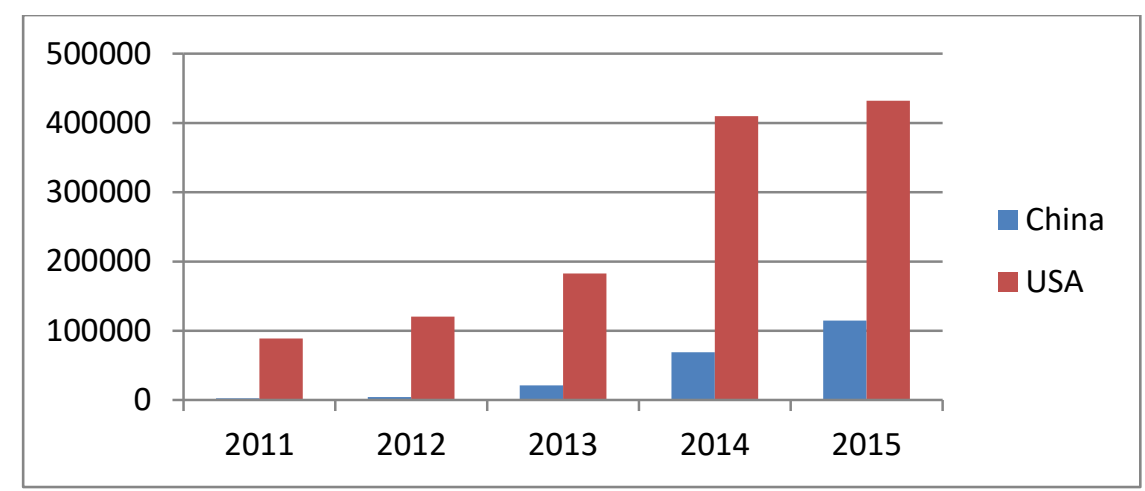

Figure 1 Comparison of Investments in Internet Medical

\section{Background}

With Internet as a carrier and information technology as a means, "Internet + " is a new form of service that is formed through combining with traditional industry services. It is changing all walks of life, constantly integrating more areas, and expanding ecology. Now, the traditional medical industry is combining with it, forming the "medical care + Internet" model. It is not just a simple sum of the two, but needs a high degree of integration and mutual penetration to form a new social form. Thus, the advantages of information technology is given play and patients' hospitalization experiences are improved. The use of Internet technology enables patients to better guided to avoid over crowdedness in hospitalization so that hospitalization efficiency is improved. By providing a variety of services, such as online or self-assisted registration, payment, collecting medicine, the number of patients queuing is reduced, the time spent is shorten. By strengthening interconnection, Internet services such as diagnosis information and cost settlement information queries are provided. By using the network, inquiry services like visit items, unit price, total cost are provided along with those like examination and inspection results. In July 2015, the State Council issued "The guidance on the active promotion of "Internet+"action" to actively promote the new model of online medical and health care. Third-party institutions were supported to build medical information sharing service platforms such as medical imaging, health records, inspection reports, and a cross-hospital medical data sharing exchange standard system was gradually established. Convenient services like online appointment diagnosis and treatment, waiting for reminders, pricing fees, diagnosis reports query were provided through the active use of the mobile Internet. Internet companies are encouraged to cooperate with medical institutions to establish medical network information platforms and to actively probe into the application of network medical and health services like extended medical advice and electronic prescriptions on the Internet.

\section{Current status in our country}

\section{Development status}

In 2014, China began to promote the standardization of medical and health information technology. Electronic health records and electronic medical records are used for residents to prevent and predict diseases. Existing resources are made full use of to strengthen telemedicine services in grass-root and remote areas. Meanwhile, remote medical service policy measures are actively formulated and promoted. The three major database resources from $50 \%$ of regional information platforms, namely databases of overall population information, electronic health file boxes and electronic medical records, are integrated and information resources from six major business systems are interconnected. Demonstration is carried out in 15 provinces and 45 large hospitals and cross-organization cross-regional sharing mechanisms of the health and medical information of residents are gradually established. In 2015the construction of the national population health information platform was sped up, and with province as a unit, the construction of provincial, municipal and county population health information platforms was planned a whole. in June 2015, the first mobile Internet-based and largest-scaled third-level first-class hospital doctor 
groups were established in China. Through mobile health cloud collaboration platform, cross-region, cross-hospital, cross-department health care collaboration was achieved. In December 2015,Wuzhen Internet hospital was put on-line. It was a new probing. Online and offline medical resources were integrated. Meanwhile, pioneer reforms such as online diagnosis and treatment, electronic medical record sharing, electronic prescriptions were initiated. It has been decided on the state level that people with serious illness will have to pay visits outside their counties for treatment until 2017 and that the establishment of national classified treatment system is to be completed in 2020.

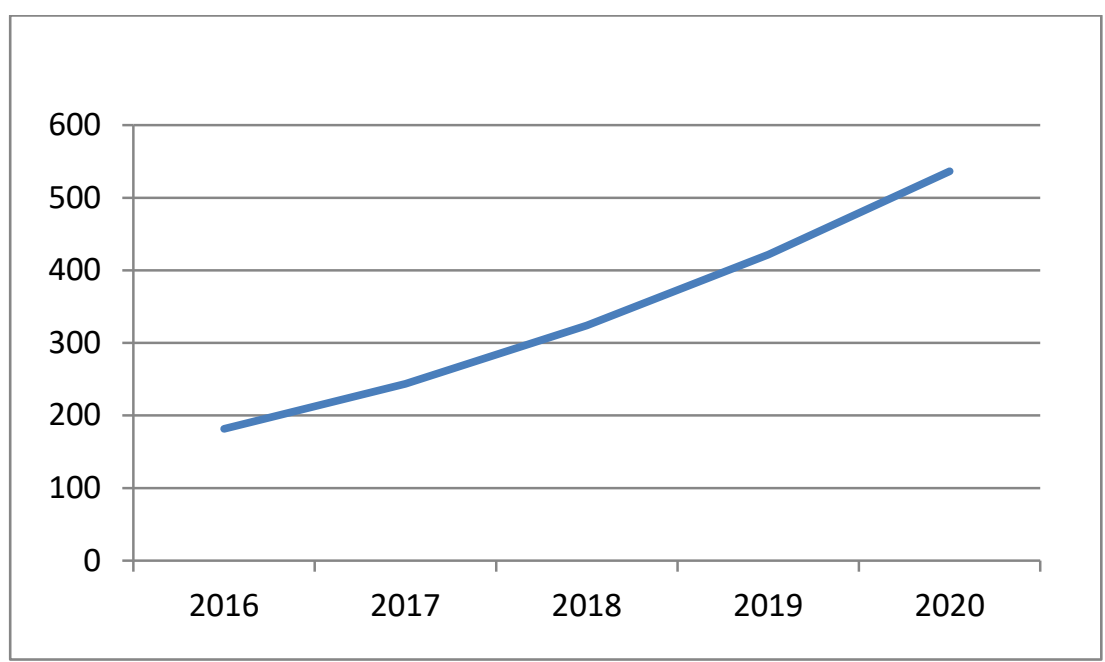

Figure 2 2015-2016 market size forecast of medical information industry

\section{Industry requirements}

(1). The aging of population

China's population aging problem: China is on the way to an aging society, and with it comes the year-after-year rise of demands for pension. In 2013, the aged population of our country was 202 million and the aging level reached $14.8 \%$. It is predicted that according to the report jointly released by BCG and Swiss Re that by 2050 the population aged 60 and over will rise to nearly 440 million. It will account for 34\%of China's total population and will result in China's entering the stage of advanced aging. The morbidity rate of chronic diseases such as coronary heart disease, hypertension, diabetes mellitus, asthma and arthritis affecting the aged population over65 is 3 to 7 times that of the population aged 15 to 45, resulting in a serious shortage of medical resources. Elderly people usually suffer from chronic diseases, and at present there is a serious information asymmetry between doctors and patients. Therefore, it is of great importance to monitor chronic diseases of the elderly population and to reduce long-term medical expenses.

(2). Uneven distribution of medical resources

China's medical resources is not only short in total amount but also uneven in distribution. Medical resources are mostly concentrated in large and medium-sized cities whereas remote rural areas is seriously short in medical resources and provide relatively low quality services. In our country, $80 \%$ of medical resources are concentrated in municipalities while $30 \%$ of them are distributed in large hospitals. It can be seen that the distribution of medical and health resources between different regions is seriously uneven and that there are also great differences in the distribution of medical and health resources in hospitals of different classes in the same area. Additionally, our country itself is limited in medical resources. This contributes to forming the concept among people that they should flock to large cities, large hospitals for hospitalization whether their illnesses are serious or not, which led to a serious waste of medical resources.

\section{Analysis of internet medical care development}

It is shown according to current medical status of our country and the international environment that Internet medical care can bring us new hospitalization concepts. Internet medical care can reconstruct traditional medical care, which is shown in the improvement of five major problems in 
traditional medical care.

\section{Achievement of disease prevention}

Internet medical care will help patients to achieve truly scientific and effective prevention and treatment of diseases before they get sick. In traditional medical care environments, patients usually do not hospitalize until their illness become obvious, but at this time their condition is very likely to be delayed, thus affecting timely treatment. In the age of internet medical care, passive treatment will turn into active disease prevention, thus nipping illnesses in the bud, and the focuses of medical services will change to chronic disease treatment and preventive health care from short-term acute medical treatment.

\section{Change the ways to hospitalize}

Internet medical care can break through the imprisonment of traditional medical care. Through online inquiries and telemedicine, cross-time-and-space configuration of quality medical resources is achieved and this spares patients from unnecessary hospitalization. Our country is unsound in medical infrastructure and unreasonable in medical resource allocation, which leads to such problems as difficulty in hospitalization on the part of patients, overcrowdedness in hospitals, low efficiency in medical care and low quality in medical services. Internet medical care helps to breakthrough limitations in regions. Through "virtualized" medical systems, patients can enjoy cross-region quality medical services. In addition, internet medical care also helps to break through imitations in time. Patients can seek advice on health from doctors whenever possible for more convenient and faster medical services. From doctors' point of view, real-time data can be acquired through continuous remote medical monitoring, the treatment of patients is sped up, and doctors' time is saved. Through real-time identification of the possible deterioration of diseases by monitoring, doctors' attention can be aroused at the earliest time.

\section{Achieve inside-hospital hospitalization experiences}

Networks have penetrated all aspects of medical services, helped to optimize patients' treatment processes inside hospitals, saved time, and improved efficiency. Firstly, registrations can be scheduled by patients. It saves time for patients to queue for registration. It enables the estimation of time for hospitalization, reduces time for patients to wait for hospitalization, improves the medical order, and relieves the over crowdedness in hospitals. Secondly, in the payment link, the trouble of queuing for payment in front of registration windows is spared through providing a variety of payment modes such as WeChat, Alipay, bank cards. Also, in the inspection report checking link, through networks patients can check in the computer or on the phone reports at the earliest time without the need to go to the hospital again to get the report printed and collect it.

\section{Change ways to buy medicine}

In the traditional medical industry, the hospital has an absolute monopoly over the use of medicine. There is a serious information asymmetry between doctors and patients. Moreover, the medicine were mostly bought during hospitalization or bought in physical retail pharmacies and it took longer time and distance to collect medicine. Modes of medicine purchases after receiving internet medical care are adopted by pharmaceutical online retailers, with B2C and $\mathrm{O} 2 \mathrm{O}$ as the main operating modes. The B2C mode allows users more convenient purchase experiences. Through internet pharmacies, medicine information can be quickly queried, prices be compared and so on. Users can take orders without going outdoors and parcels can also be delivered express to your home. Through the rapid logistical distribution by physical retail pharmacies, the $\mathrm{O} 2 \mathrm{O}$ mode attempts to complete medicine distribution within one hour to bring customers more convenient purchase experiences. Meanwhile, through the reduction of intermediate circulation, there is a greater discount of prices of online drugs as compared with offline drugs. It allows users to buy cheaper drugs. It is also a factor that improves online shopping experiences and attracts users to buy medicine online. In the future the separation of prescription from pharmacy will become increasingly clear. There will be substantial growth in the species of online medical sales. These will change customers' medicine purchase habits and bring them more comfortable experiences.

\section{Improve doctor-patient relationship}

Optimizing doctor-patient docking mechanisms and promote the doctor-patient communications 
enable the value of doctors to be maximized and services to be optimized. In recent years, such behaviors as patients' blackmailing, abusing, assaulting, and siegeing hospitals out of their dissatisfaction with medical staffs frequently occurred, hence the continuous escalation of the conflicts between doctors and patients. The involvement of the Internet has improved the relationship between doctors and patients. Internet medical care allows online dialogues with patients to be conducted by doctors about such aspects as health management, self-diagnosis and so on. Thus the needs of patients can be fully listened for, and communications between doctors and patients can be promoted. Internet medical care has broken the limits of fixed occupation and created ways to increase income for doctors on the edge of policies. Online diagnosis have eased the asymmetry of medical resources. Excellent doctors can make use of their fragmented time to help more patients who have originally failed to get the service and gain fame and word of mouth at the same time. Internet medical care is a combination of online and offline services and gives patients warmer experiences. The internet will turn the traditional "hospital-and-doctor-centered" medical service mode to the "patient-centered" medical service mode. Through the optimization of service processes, internet medical care will provide patients with humanistic care and services like referral, interpretation, counseling, thus bringing more convenience and warmth to patients during hospitalization.

\section{Summary}

Internet medical care will bring with it new health concepts, technology and means. Through the effective combination of "Internet+" with doctors and medical institutions, the traditional medical mode of "patients going to hospital for minor or serious illnesses and doctors sitting at fixed places to see patients" will be changed, medical and health service systems with online medical care as the representative will optimized, medical services will be extended to before and after hospitalization from during hospitalization, and convenient services will be provided to patients and doctors respectively. Medical care + Internet is an inevitable trend. For the pharmaceutical industry, there are many opportunities to explore and probe into. Seize the opportunity and believe that, following the footsteps of the Internet, there will be greater room for development in the future.

\section{Acknowledgements}

This work was supported in part by the technology projects of Suzhou science and technology development project (NO.SYSD2015014) and Science and Technology Development Project of Changshu Grant (No.CS201503)

\section{References}

[1]Jiang X W. Analysis and Thought of Developing Nationa l Primary Medical Care System in China[J]. Chinese Primary Health Care, 2013.

[2]Zhou, Fen, H. Chen, and J. Lin. "Analysis of Efficiency and Fairness of the Current Basic Medical Service in China." Heilongjiang Medical Journal (2013).

[3]Jones, French Allan. "The Analysis of Patient Status Following Substance Abuse Treatment and Utilization of Medical Care." Plos Genetics 6.6(2010):773-777.

[4]Chen, Guo Yong, et al. "Analwysis of status and influence factors of literacy of basic medical care of the urban and rural residents in China." Chinese Journal of Health Education (2015).

[5]Xiao-Qin, Y. E., Z. J. Guan, and K. Meng. "Analysis of the Research Status of Regional Medical Association Analysis in China Based on Bibliometrical Method." Chinese Primary Health Care (2016). 